\title{
A new polarimeter scheme based on solid state semiconductors
}

\author{
Un nuevo esquema para polarímetros \\ basado en semiconductor de estado sólido
}

\author{
Heiner Castro Gutierrez ${ }^{1}$
}

Recibido 10 de octubre de 2011, aceptado 28 de agosto de 2012

Received: October 10, $2011 \quad$ Accepted: August 28, 2012

\begin{abstract}
RESUMEN
Un nuevo esquema de polarímetro es sugerido usando semiconductores de estado sólido. La nueva aproximación está basada en la modulación sobre las intensidades de los rayos difractados a través de una rejilla quiral de dos dimensiones reportado recientemente. Será demostrado que al menos cuatro mediciones de intensidades de rayos difractados no equivalentes son necesarios para estimar el estado de polarización del rayo incidente. El azimut del rayo incidente es variado, rotando un lente polarizador lineal montado en un motor paso a paso. La intensidad de cuatro rayos difractados es medida por medio de una pequeña pantalla, una cámara CCD y algunos algoritmos corriendo en un computador. El software de desarrollo LabVIEW fue usado para controlar el hardware y presentar los resultados. Matlab fue utilizado para calcular las intensidades de los rayos difractados y así computar el azimut y la elipticidad del rayo incidente. Aunque en teoría los dos parámetros, el azimut y la elipticidad, pueden ser estimados, los experimentos muestran que solo la estimación del azimut es precisa. Mientras que la elipticidad no puede ser estimada con precisión. El error en la estimación del azimut depende de las variaciones de potencia del rayo incidente. La estimación del azimut fue encontrada correcta entre los grados $[0,140)$ y $(150,180]$. Los grandes errores en el azimut encontrados entre 140 y 150 grados ocurren por razones desconocidas.
\end{abstract}

Palabras clave: Polarización, azimut, elipticidad, intensidad de la luz, rejillas quirales.

\begin{abstract}
A new kind of polarimeter scheme is suggested using solid state semiconductors. The new approach is based on the modulation over the intensities of the diffracted beams through a two-dimensional chiral grating, reported recently. It will be demonstrated that at least four intensity measurements of no equivalent diffracted beams are needed in order to estimate the polarization state of the incident beam. The incident beam azimuth was varied by routing a linear polarizer lens mounting in a stepped motor. The intensities of four diffracted beams were measured using a screen, a CCD camera and some algorithms running in a computer. The LabVIEW development environment software was used for controlling the hardware and for presenting the results. MATLAB@ was used for calculating the intensities of the diffracted beams and computing the azimuth of the incident beam. Although both the azimuth and ellipticity should be estimated, the experiments show that only the azimuth estimation yields accurate results. The ellipticity cannot be estimated with precision. The error on the azimuth estimation depends on the variation in the power of the incident beam. It was found that the azimuth estimation is accurate between $[0,140)$ and $(150,180]$ degrees. The cause of huge errors in the azimuth found between 140 and 150 degrees are kept unknown.
\end{abstract}

Keywords: Polarization, azimuth, ellipticity, light intensity, chiral gratings.

1 Facultad de Ingeniería. Programa de Ingeniería Electrónica. Universidad del Magdalena. Carrera 32 № 22-08 Santa Marta. Colombia. E-mail: hcastro@unimagdalena.edu.co 


\section{INTRODUCTION}

All currently available commercial polarimeters operate by measuring the difference in the relative intensity of the incident light after it has passed through one or more filters. These filters include linear polarizers oriented at $0^{\circ}, 90^{\circ}, 45^{\circ}$ and $135^{\circ}$ to the vertical, left and right-handed circular polarizers and neutral density filters. These intensity measurements are then used to calculate the Stokes' parameters $\left(\mathrm{S}_{0}\right.$, $S_{1}, S_{2}$, and $S_{3}$ ) from which the azimuth angle and the ellipticity of the input beam can be determined [1]. The main disadvantage of current polarimeters is that they require either light splitters or moving parts in order to analyse the beam intensity to obtain the Stokes' parameters.

Certain research work developed recently on the optic properties of the $2 \mathrm{D}$ chiral gratings conducts the idea of a new type of polarimeters. The new polarimeter proposal is based in solid state technology. Therefore, no mechanical or splitter disadvantages are associated. It will be shown that the intensity and polarisation state of the diffracted beams from these $2 \mathrm{D}$ chiral gratings shows a strong modulation as a function of the polarization state of the incident beam. Besides, it will be demonstrated that this dependency is linear and as a result it complies the principle of superposition. Furthermore, the system can be modelled using transfer matrices to represent the intensities of the diffracted beams as a function of the polarization state of the incident beam. Thus, it will be possible calculate the input polarization state by measuring light intensity only. Moreover, some recent papers states that this approach can lead to a multifrequency polarimeter system. That is to say, polarimeters capable of measuring the polarization state of two or more optical wavelengths simultaneously [2], something impossible to achieve with available polarimeters. This work is the first practical approximation to probe that such polarimeter scheme could work.

\section{THEORETICAL ANALYSIS}

Recently, it has been reported a strong linear dependency on the state of polarization of the incident beam for both the polarization state and the intensity of diffracted beams through two-dimensional (2D) chiral structure $[3,4]$. In this case 2D chiral feature is defined as "a planar surface that does not possess a line of symmetry within the plane of the patterned surface, and where if the surface pattern is mirror-reflected about any line in the plane of the surface. The resulting pattern cannot be brought into perfect congruence with the original surface pattern by any rotation about any axis normal to the plane, or any linear translation within the plane, or any combination of the two" [5]. A picture of the Planar Chiral Material (PCM) used for the experiments is shown in Figure 1.

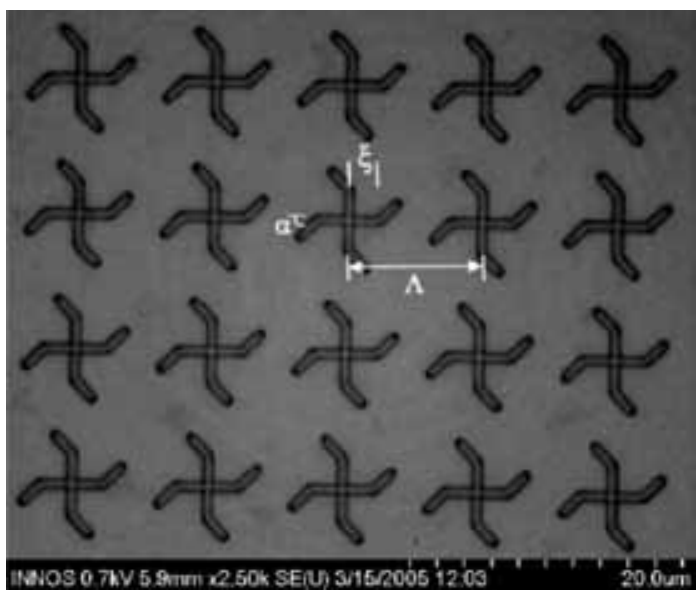

Figure 1. Planar Chiral Material used in the project.

The sample was fabricated using electron beam lithography and reactive ion etching. The sample was engineered in silicon nitride $\left(\mathrm{Si}_{3} \mathrm{~N}_{4}\right)$ layers and is supported on $1 \mathrm{~mm}$ thick fused quartz wafer [3]. Fused quartz was employed because it is transparent at $633 \mathrm{~nm}$ which is the wavelength used for the experiments. Each chiral design consists of gammadions in a planar tiling configuration with bending angles $(\alpha)$ of $45^{\circ}$, arm segment $(\xi)$ of $1.8 \mu \mathrm{m}$ and gammadion pitch $(\Lambda)$ about $5 \mu \mathrm{m}$ (see Figure 1 for details).

In order to simplify the calculation of the polarization state of the input beam based on the intensities of the diffracted beams the Jones calculus approach will be used in this paper. This approach allows easy matrix calculation of complicated polarization problems [6]. Any state of polarization can be represented by the use of the Jones vector $J$ [7]:

$$
J(\varphi, \eta)=p\left[\begin{array}{l}
1 \\
Z
\end{array}\right]
$$

where $\varphi, \eta$ are the azimuth and the ellipticity respectively and $p$ and $Z$ are described by 


$$
\begin{gathered}
p=\cos (\varphi) \cos (\eta)+i \sin (\eta) \sin (\varphi) \\
Z=r e^{i \delta}
\end{gathered}
$$

where the amplitude factor $r$ and the phase $\delta$ are related by

$$
\begin{gathered}
r^{2}=\frac{\tan ^{2}(\Delta \varphi)+\tan ^{2}(\eta)}{1+\tan ^{2}(\Delta \varphi) \tan ^{2}(\eta)} \\
\tan (\delta)=\frac{\tan (\eta)\left[1+\tan ^{2}(\Delta \varphi)\right]}{\tan (\Delta \varphi)\left[\tan ^{2}(\eta)-1\right]}
\end{gathered}
$$

The azimuth rotation angle $\Delta \varphi$ is the difference between the azimuth angle of the input beam $\left(\varphi_{0}\right)$ and the azimuth angle of the diffracted beam $\left(\varphi_{1}\right)[7]$

$$
\Delta \varphi=\varphi_{1}-\varphi_{0}
$$

Note that in [2] equations (4) and (5) also include the ellipticity rotation $\Delta \eta=\eta_{1}-\eta_{0}$. However, at the time of the experiments here presented, the mentioned paper was not published. As stated above, the polarization and intensity of the diffracted beams from dielectric chiral gratings response linearly to changes in the polarization of the incident beam. Therefore, the change in the polarization state can be modeled using Jones matrix calculus and then written in the form [7]

$$
\mu_{10} J_{1}=A \cdot J_{0}
$$

where $J_{0}$ and $J_{1}$ are the Jones vectors for the input and output state respectively. The parameter $\mu_{10}$ is complex and represents the relative phases and amplitudes of the output and input states. $A$ is the transfer matrix of the system and it is particular for each diffracted beam and can be expressed as

$$
A=\left[\begin{array}{cc}
1 & -Z_{1} Z_{2} \\
Z_{1} & Z_{0}
\end{array}\right]
$$

where the complex terms $\mathrm{Z}_{0}, \mathrm{Z}_{1}$ and $\mathrm{Z}_{2}$ can be determined by taking measurements of the output polarization state $J_{1}$ for three different linearly polarized input states and taking into account equation (3) to (6). The parameter $Z_{1}$ can be derived by measuring the output polarization state $\left(\varphi_{1}\right.$ y $\eta_{1}$ ) for a linear horizontally polarized input state $\left(\eta_{0}=0^{\circ}\right.$ and $\left.\varphi_{0}=0^{\circ}\right)$ and substituting the measured values into equations (3) to (6). Similarly, $Z_{2}$ can be found by measuring the output polarization state for a linear vertically polarized input state $\left(\eta_{0}=0^{\circ}\right.$ and $\varphi_{0}=90^{\circ}$ ) and substituting the measured values $\left(\varphi_{1}\right.$ y $\left.\eta_{1}\right)$ into the same group of equations. The term $Z_{0}$ is determined by first, finding $Z_{3}$ using the same method described above, for an input state which is a linear superposition of the inputs used to find $Z_{1}$ and $Z_{2}$. That is to say, $Z_{3}$ should be a linear polarized state with azimuth between 0 and 90 degrees $\left(0<\varphi_{3}<90^{\circ}\right)$ [7]. Secondly, $Z_{0}$ can be calculated from

$$
Z_{0}=\frac{Z_{1}+Z_{1} Z_{3} \tan \left(\phi_{3}\right)+\tan \left(\phi_{3}\right)-Z_{3}}{Z_{3} \tan ^{2}\left(\phi_{3}\right)+Z_{2} Z_{3} \tan \left(\phi_{3}\right)+\tan \left(\phi_{3}\right)-Z_{2} \tan ^{2}\left(\phi_{3}\right)}
$$

Thus, only three polarization state measurements of the diffracted beams are needed in order to find the $\mathrm{Z}$ parameters and complete the transfer matrix $A$. The intensity $I$ of the each diffracted beam is also linearly related with the polarization state of the input $[2,7]$ and is given by

$$
I=I_{0}\left[\begin{array}{l}
\alpha_{0}+\alpha_{1} \cos (2 \varphi) \cos (2 \eta)+ \\
\alpha_{2} \sin (2 \varphi) \sin (2 \eta)+\alpha_{3} \sin (2 \eta)
\end{array}\right]
$$

where $I_{0}$ is the power of the incident beam and the factors $\alpha_{0}, \alpha_{1}, \alpha_{2}$ and $\alpha_{3}$ are given by

$$
\begin{gathered}
2 \alpha_{0}=1+x_{1}^{2}+y_{1}^{2} \\
+\left(x_{0}^{2}+y_{0}^{2}\right)\left(1+x_{2}^{2}+y_{2}^{2}\right) \\
2 \alpha_{1}=1+x_{1}^{2}+y_{1}^{2} \\
-\left(x_{0}^{2}+y_{0}^{2}\right)\left(1+x_{2}^{2}+y_{2}^{2}\right) \\
\alpha_{2}=x_{0}\left(x_{2}-x_{1}\right)-y_{0}\left(y_{1}+y_{2}\right) \\
\alpha_{3}=y_{0}\left(x_{2}-x_{1}\right)+x_{0}\left(y_{1}+y_{2}\right)
\end{gathered}
$$

where the terms $x_{0}, x_{1}$ and $x_{2}$ are the real parts of the complex terms $\mathrm{Z}_{0}, \mathrm{Z}_{1}$ and $\mathrm{Z}_{2}$ respectively; and $y_{0}, y_{1}$ and $y_{2}$ are the respective imaginary parts [2, 7]. Now, if equation (10) is rewritten in the form of 


$$
I=\alpha_{0} I_{0}+\alpha_{1} X+\alpha_{2} Y+\alpha_{2} W
$$

where

$$
\begin{gathered}
X=I_{0} \cos (2 \varphi) \cos (2 \eta) \\
Y=I_{0} \sin (2 \varphi) \sin (2 \eta) \\
W=I_{0} \sin (2 \eta)
\end{gathered}
$$

Equation (15) has four unknown variables e.g. $I_{0} X$, $Y$ and $W$. Therefore, four intensity measurements of four diffracted beams are required in order to find the unknowns. Using a matrix representation the whole system can be written as

$$
\left[\begin{array}{cccc}
\alpha_{01} & \alpha_{11} & \alpha_{21} & \alpha_{31} \\
\alpha_{02} & \alpha_{12} & \alpha_{22} & \alpha_{32} \\
\alpha_{03} & \alpha_{13} & \alpha_{23} & \alpha_{33} \\
\alpha_{04} & \alpha_{14} & \alpha_{24} & \alpha_{44}
\end{array}\right]\left[\begin{array}{c}
I_{0} \\
X \\
Y \\
W
\end{array}\right]=\left[\begin{array}{c}
I_{1} \\
I_{2} \\
I_{3} \\
I_{4}
\end{array}\right]
$$

where $\alpha_{\mathrm{nj}}$ denotes the $\mathrm{n}$-th $\alpha$ parameter for the $\mathrm{j}$-th beam and $I_{1}, I_{2}, I_{3}$ and $I_{4}$ are the measured intensities for four diffracted beams respectively. Moreover, once the $\alpha$ parameters have been found for a particular set of diffracted beam the solution of equation (19) can be used for finding the state of polarization of the input beam.

\section{RESULTS}

\section{Experiment description}

The complete experiment arrangement is illustrated in Figure 2 where all used components can be seen. The experiments were performed with two linear polarizer, one is the first lenses and the second was mounted in the stepper motor (NanoRotator in Figure 2.). Besides, one circular polarizer lens was used between the two linear polarizer lenses. At the right of the stepper motor the chiral material sample was positioned and beyond the screen and the CCD camera.

The light source was a HeNe laser operating at $633 \mathrm{~nm}$. The laser was first passed through a liner polarizer, after across a circular polarizer and finally through a linear polarizer in order to come the beam back into a linear polarization state. All that lenses are required to ensure a changeable polarization state. These changes will be done by the last linear polarizer, mounted into a stepper-motor. Rotating the stepper-motor the azimuth of the beam is also rotated. The stepper motor used was the NanoRotator $360^{\circ}$ model NR360S by Thorlabs $®$ and controlled by an APT System (Stepper motor controller in Figure 2). This stepper motor can achieve a step in degrees of 360/25600. The laser beam, after passing along the lens system, hits the chiral material sample and the diffracted beams are projected on the screen. A picture then can be taken using the Thorlabs ${ }^{\circledR}$ CCD camera DC310C, which is connected to a computer

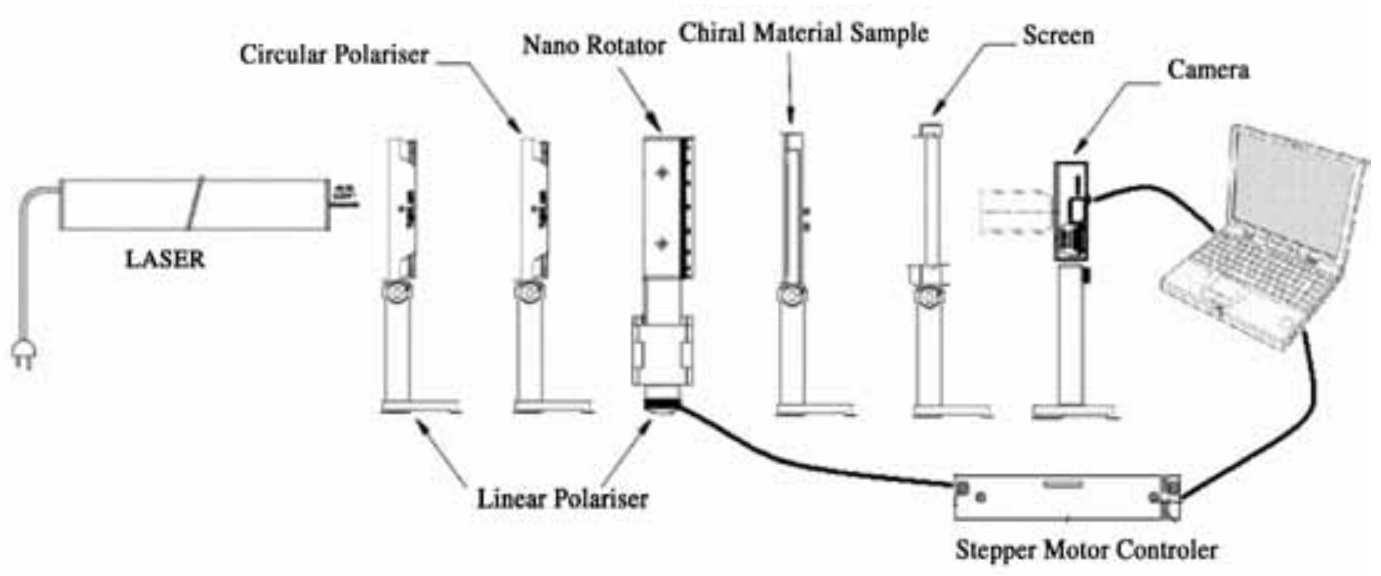

Figure 2. Hardware arrangement. 
via a FireWire port. The picture analysis algorithm is executed into the computer using MATLAB $\odot$. The motor and the camera are also controlled by the computer using LabVIEW@ environment software. Several tests and experiments were accomplished using this arrangement. The results of some of them will be presented in the next section.

\section{Discussion of results}

All power measurements were performed using the Thorlabs (®) PM120 Power Meter System calibrated in the last periodic device maintenance process. The very first power measurements were analyzed in order to choose the four beams required to determine the input polarization state. First, the output power of the first order beams was registered and the results are shown in Figure 3.

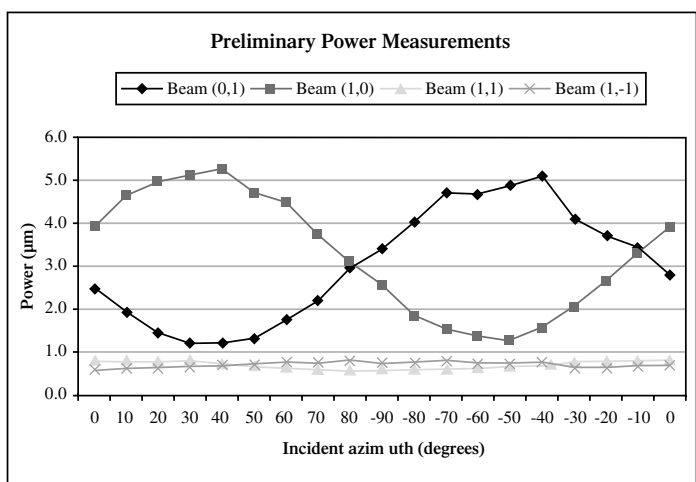

Figure 3. First order beams power output. The azimuth angle is represented from $-90^{\circ}$ to $90^{\circ}$. It means that the azimuth values from $-90^{\circ}$ to $0^{\circ}$ correspond to angles from $90^{\circ}$ to $180^{\circ}$.

The modulation of the output power over the input azimuth can be observed in Figure 3, attesting the results found in $[2,4,7]$. It can be noticed that the beams $(1,1)$ and $(1,-1)$ are much weaker than beams $(0,1)$ and $(1,0)$. A better view of the weakest beams is displayed in Figure 4. It can be observed that the modulation is not as regular as in beams $(1,0)$ and $(0,1)$ due to unevenness in the laser power, especially at the beam $(1,-1)$. The laser power stability will be discussed later in this paper.

Second order beams were tested for comparing their power and modulation regularity against the power of the first order beams. It was found that beams $(2,0)$ and $(2,1)$ exhibit a regular modulation

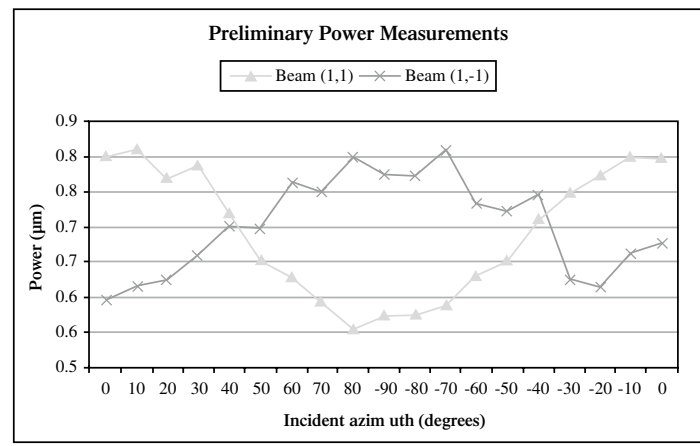

Figure 4. Output power for the beams $(1,1)$ and $(1,-1)$

over changes in the azimuth of the input beam. Besides, the power of these beams are in the same order of magnitude of the beams $(0,1)$ and $(1,0)$. Due to this, these groups of beams were chosen to perform the final experiments. Figure 5 shows the selected beams to execute the experiments. The output power modulation observed in Figure 5 also supports the results found in $[2,4,7]$.

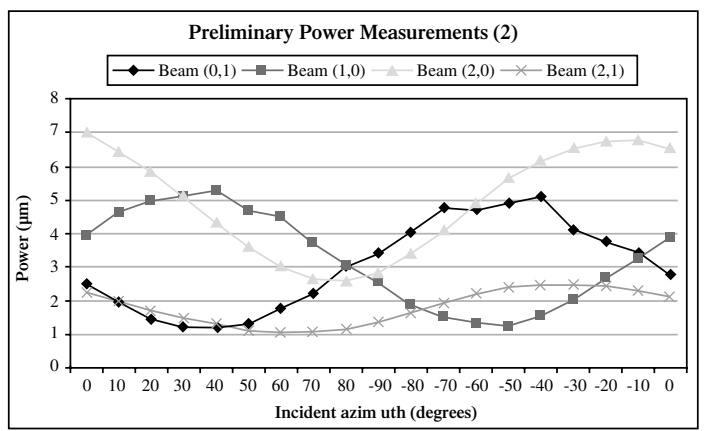

Figure 5. Selection of beams to perform the experiments.

The power stability of the incident beam is very important for the experiment since any change in the power of the incident beam can lead unexpected alterations in the output power of the diffracted beams. Unfortunately, it is not possible to measure the power of the incident beam during the experimentation process, because the input beam would be obstructed by the sensor of the power meter. Therefore, separate measurements for both the input power and one diffracted beam power were performed in order to investigate any possible relationship between these two magnitudes.

It was observed that for the first 30 minutes of functioning of the laser, the power is very unstable, perhaps owing to the heating up process of the 
device. Due to this, all the experiments were accomplished after at least one hour of starting the laser. Even after around 1 hour since starting, the power of the incident beam is not properly stable. The average power of the input beam and his respective standard deviation were found around $0.75 \mathrm{~mW}$ and $0.02 \mathrm{~mW}$ respectively, for a percentage of change about $2.7 \%$. The anterior measurements were done over 4 hours of laser functioning. This oscillation may produce instability in the power of the diffracted beams, which would insert error in the measurements of intensities. For the beam $(2,0)$ it was found an average power and a standard deviation around $6.2 \mu \mathrm{W}$ and $0.6 \mu \mathrm{W}$ respectively for a percentage of change about $9.7 \%$. As a result, small changes in the power of the incident beam $(2.7 \%)$ can produce larger changes in the power of the diffracted beams $(9.7 \%)$.

The Azimuth estimation of the incident beam is shown in Figure 6 along with the ideal response of the system. It can be seen that the system follow the ideal line, except for angles between 140 and 150 degrees. The incident azimuth is given by the position of the stepper motor (see Figure 2) which is changed every 2 degrees. The experiment was repeated several times and the results were always the same: the azimuth estimation follows the expected behavior excluding angles between 140 and 150 degrees. The reason of this behavior remains unknown.

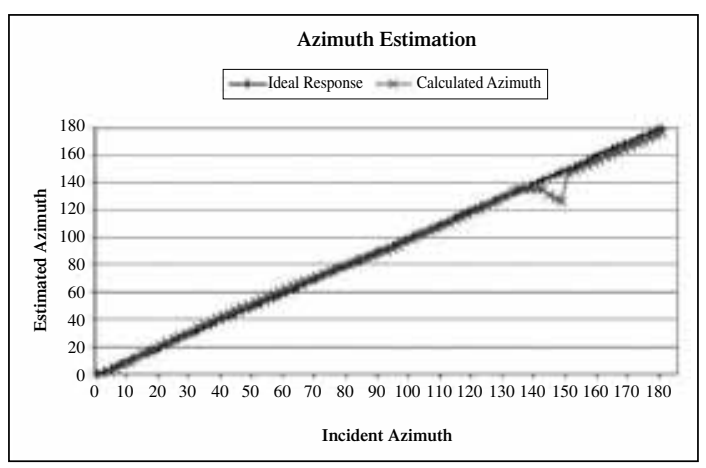

Figure 6. Comparison between the ideal and real azimuth response

The absolute error calculated as the absolute difference between the computed azimuth (algorithm output) and the azimuth of the incident beam (stepper-motor position) is illustrated in Figure 7. It can be seen that the error is not constant and varies throughout the inputs. Moreover, it can be noticed that the absolute error can be as big as 4 degrees and as small as approximately 0 degrees. Presumably, this error is not constant due to the instability of the incident beam power.

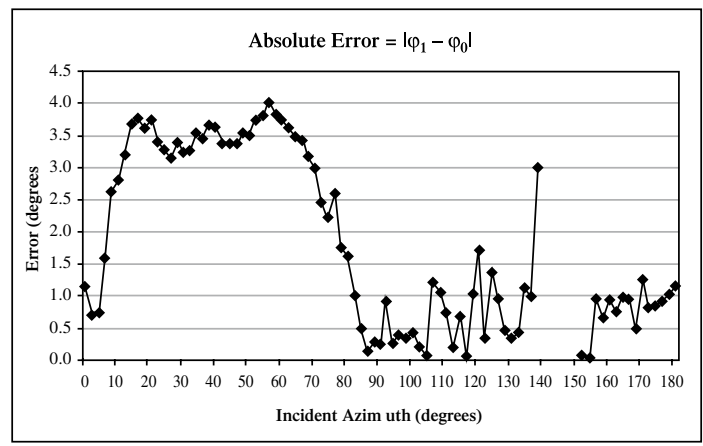

Figure 7. Absolute error for the incident azimuth calculated for the system. Errors from $140^{\circ}$ to $150^{\circ}$ have been eliminated purposefully, because these errors are very large.

More experiments were performed and the outcomes were very analogous: the absolute error varies between approximately 0 and around 4 degrees. On the other hand, comparing the results of all these experiments, it can be seen that the registered errors for equal inputs were very dissimilar. Consequently, the error does not depend upon the incident azimuth. Presumably, this behavior is due to the power oscillations in the incident beam. In order to investigate the latter a series of experiments were accomplished where the incident azimuth was not varied. The obtained results were very conclusive: the standard deviation of the registered data were relatively scattered. The minimum deviation was about $0.05^{\circ}$ and the maximum was around $0.37^{\circ}$. For instance, for an incident azimuth of $30^{\circ}$ the standard deviation was as small as $0.07^{\circ}$ and the maximum was as high as 0.27 . All these tests were conducted under the same conditions of the experiments presented above. Table 1 shows the results of these experiments. It can be noticed that there is no correlation between the azimuth of the incident beam and the absolute error as well as there is no relation between the incident azimuth and the deviation of the estimated azimuth either.

Finally, the solution for equation (19) should lead to find both azimuth and ellipticity. Although for the 
Table 1. Results of multiple experiments for equal incident azimuth.

\begin{tabular}{|c|c|c|c|}
\hline Az=0 & Average & Error (abs) & Std. dev. \\
\hline Experiment 1 & $1.32^{\circ}$ & $1.32^{\circ}$ & $0.10^{\circ}$ \\
\hline Experiment 2 & $-1.20^{\circ}$ & $1.20^{\circ}$ & $0.09^{\circ}$ \\
\hline Experiment 3 & $3.25^{\circ}$ & $3.25^{\circ}$ & $0.07^{\circ}$ \\
\hline Experiment 4 & $-0.05^{\circ}$ & 0.05 & $0.26^{\circ}$ \\
\hline Az=30 & Average & Error (abs) & Std. dev. \\
\hline Experiment 1 & $33.40^{\circ}$ & $3.40^{\circ}$ & $0.22^{\circ}$ \\
\hline Experiment 2 & $28.50^{\circ}$ & $1.50^{\circ}$ & $0.14^{\circ}$ \\
\hline Experiment 3 & $26.90^{\circ}$ & $3.10^{\circ}$ & $0.09^{\circ}$ \\
\hline Experiment 4 & $33.96^{\circ}$ & $3.96^{\circ}$ & $0.27^{\circ}$ \\
\hline Az=60 & Average & Error (abs) & Std. dev. \\
\hline Experiment 1 & $64.17^{\circ}$ & $4.17^{\circ}$ & $0.37^{\circ}$ \\
\hline Experiment 2 & $56.30^{\circ}$ & $3.70^{\circ}$ & $0.15^{\circ}$ \\
\hline Experiment 3 & $57.50^{\circ}$ & $2.50^{\circ}$ & $0.29^{\circ}$ \\
\hline Experiment 4 & $63.20^{\circ}$ & $3.20^{\circ}$ & $0.35^{\circ}$ \\
\hline Az=90 & Average & Error (abs) & Std. dev. \\
\hline Experiment 1 & $89.20^{\circ}$ & $0.80^{\circ}$ & $0.05^{\circ}$ \\
\hline Experiment 2 & $89.75^{\circ}$ & $0.25^{\circ}$ & $0.13^{\circ}$ \\
\hline Experiment 3 & $91.10^{\circ}$ & $1.10^{\circ}$ & $0.08^{\circ}$ \\
\hline Experiment 4 & $89.60^{\circ}$ & $0.40^{\circ}$ & $0.19^{\circ}$ \\
\hline
\end{tabular}

azimuth estimation the experiments show a relative good response, this is not the case for ellipticity estimation. The results over this magnitude are not accurate. The ellipticty was let constant over all the experiments, but the obtained data are spread over all the interval range e.g. 0 to 180 degrees. Accordingly, the results over this magnitude are inconclusive.

\section{CONCLUSIONS}

The theory needed for producing a new kind of polarimeter was expounded. The new polarimeter proposal consists in measuring the intensities of diffracted beams through a dielectric planar (2D) chiral material. It was studied that at least four intensities of different diffracted beams are required to solve a system of equation in order to determine the polarization state of the incident beam.

The hardware employed to perform the experiments was described: two Thorlabs ${ }^{\circledR}$ power meter systems, one Thorlabs ${ }^{\circledR}$ Stepper Motor and one Thorlabs ${ }^{\circledR}$ CCD camera. All these devices were controlled using the development environment software LabVIEW@. The azimuth of the incident beam was varied rotating a linear polarizer lent mounted into the stepper-motor.

The input azimuth was predicted; hence the accuracy of the system could be tested. The evidence shows that the series of achieved results are repetitive, since similar outcomes were accomplished after multiple iterations were performed. The experiments showed a prediction in the azimuth of the incident beam relatively accurate in the interval of $[0,140)$ $\mathrm{U}(150,180]$ degrees. The tests demonstrated an anomalous behaviour in the interval $[140,150]$ degrees. There is no scientific evidence of the reasons for this behavior. Furthermore, future work should be concentrate on this matter. The absolute error achieved in all the experiments varies from 0.03 to 4.17 degrees. This error does not depend on the measurement method or on the devices used. Moreover, different errors are measured for the same point when the experiments are repeated. It is thought that this instability is due to the laser power stability. Accordingly, the experiments should be tested with a very stable source of light.

Future progress in this work should focus on examining deeply possible causes of inconsistent response, and possible solutions as well, for an incident beam with azimuth between 140 to 150 degrees. More work should concentrate on the estimation of the ellipticity which was not reached in this work. One solution to these issues can be the selection of more than four beams for the solution of the equations. Another can lead to test several combinations of beam sets to choose the best array in order to achieve more accurate results. For reducing the deep error dependency on the power oscillation of the incident beam the calculation of the ratio between the input and output intensity should be attempted. For better results, one set of lenses to project diffracted beams direct on the sensor of the CCD camera should be probed. This might lead to more accurate intensity measurements.

\section{ACKNOWLEDGMENTS}

I would like to acknowledge the help and support of the people of Thorlabs and LabVIEW for his invaluable favors with the drivers and the software issues. 


\section{REFERENCES}

[1] M. Born and E. Wolf. "Principles of Optics". Cambridge University Press. Seventh edition, pp. 630-632. Cambridge. United Kingdom. 1999. ISBN-10: 0521642221. ISBN-13: 978-0521642224.

[2] A. Potts, W. Zhang and D.M. Bagnall. "A new polarimeter based on optical nonreciprocity in gratings with two-dimensional chirality". Applied Physics B: Lasers and Optics. Vol. 99, pp. 679-693. June, 2010. ISSN 1741-3567 DOI: $10.1088 / 1464-4258 / 6 / 2 / 00$.

[3] A. Potts, W. Zhang and D.M. Bagnall. "Giant optical activity in dielectric planar metamaterials with 2D chirality". Journal of Optics A: Pure and Applied Optics. Vol. 8, pp. 878-890. August, 2006. ISSN 2040-8986. DOI:10.1088/1464-4258/8/10/00.
[4] W. Zhang, A. Potts, A. Papakostas and D.M. Bagnall. "Intensity modulation and polarization rotation of visible light by dielectric planar chiral metamaterials". Applied Physics Letters. Vol. 86, pp. 2319051-231905-3. April, 2005. ISSN DOI: 10.1063/1.1944211.

[5] A. Papakostas, A. Potts, D.M. Bagnall, S.L. Prosvirnin, H.J. Coles and N.I. Zheludev. "Optical Manifestations of Planar Chirality". Physical Review Letters. Vol. 90, pp. 107404-1107404-4. March, 2003. ISSN 1079-7114. DOI: 10.1103/PhysRevLett.90.10740.

[6] D.H. Goldstein. "Polarized Light". CRC Press. Second edition, pp. 211-23. New York. USA. 2003. ISBN-10: 082474053X.

[7] A. Potts, W. Zhang and D.M. Bagnall. "Nonreciprocal diffraction through dielectric gratings with two-dimensional chirality". Physical Review A. Vol. 77, pp. 43816-143816-14. April, 2008. DOI: 10.1103/ PhysRevA.77.04381. 